\title{
Elimination of root canal filling material from the root and the bone
}

\author{
Vladimir Panov \\ Department of Conservative Dentistry and Oral \\ Pathology, Faculty of dental medicine, Medical \\ University -Varna;
}

\begin{abstract}
We present a case of a 43 years old male with mandibular central incisors trauma which dates back to 1985, i.e. 34 years before the current study. At that time the patient is 9 years old and the teeth are with incomplete root development. An endodontic treatment of 31 and 41 is conducted, and followed by phisioterapy. The patient has no complaints, and the first radiographic investigation is made 12 years later, in 1997. Radiograph reveals teeth with non-exact endodontic treatment - canals are over-filled and short-filled in the same time. Overextension of filling material into the surrounding bone tissues with a resorption around it on 3-4 $\mathrm{mm}$ apically to 31 is confirmed. Voids are present in the root filling and between root filling and root canal walls. There is a loss of the alveolar bone between 31 and 41.
\end{abstract}

An endodontic revision with apical surgery is recommended, but no treatment is executed. We observe a complete absorption of over-filled canal filling material from the bone for a period of 25 years. At the 34 year we see only a slight lightening at the place of the overfilled material and an almost complete lack of canal filling of 31 .

34 years after the treatment, the patient has not received any treatment and has never had any complaints. The teeth are still restored with the temporary obturation from 1985. Over time, root canal filling is eliminated not only from the bone, but from the root canals.

Keywords: canal filling material, overextension, complications, radiographs, elimination 


\section{Introduction}

The functionality and survival of endodontically treated teeth is often the most important aspect of endodontic treatment, which is an effective method for maintaining natural teeth (1). According to some authors, five-year survival rate of endodontically treated teeth is about $90 \%$ (2). Another large-scale study of over 1.6 million patients with root canal therapy shows $97 \%$ retention of these teeth 8 years following the procedure. Most of the undesired events like re-treatment, apical surgery or extraction occur during the first 3 years after the initial endodontic treatment (3). Endodontically treated teeth are prone to extraction mainly due to non-restorable destruction, improper fit of the crown margins that encircles the tooth, which leads to the ingress of bacteria, and to a lesser extent to endodontic-related reasons such as endodontic failure, root fracture, perforation (4).

In most of the cases, endodontic materials should be limited into root canals without extension in surrounding tissues during root canal treatment. Overextension of materials may take place through overinstrumentation, perforation of the root canals, incomplete tooth formation in children, allowing passage of dressing agents, sealers, and filling materials to adjacent structures. In this situation, the patient may experience complications such as pain or tissue necrosis (5). The complications may be even more severe when the overextended material reaches the mandibular canal, maxillary sinus, mental foramen or incisive foramen, resulting in neurologic disturbances, paraesthesia, hyperaesthesia or dysaesthesia, or mechanical and chemical irritation of the adjacent tissue (6).

When patients complain of neurological disturbances after root canal treatment, radiographic images should be taken to verify possible overextension of materials and to determine the location of the overfilled materials (7).

\section{Case report}

We present a case of a 43 years old male with mandibular central incisors trauma which dates back to 1985 , i.e. 34 years before the current study. At that time the patient is 9 years old and the teeth are with incomplete root development. Several weeks after the trauma, there is purulent inflammation and teeth 31 and 41 are with elevated mobility. An endodontic treatment is conducted in about 15 appointments for two months in First City Hospital-Sofia. Root canals are obturated and 10-15 procedures of physical therapy (probably electrophoresis) are applied. Teeth are closed with temporary filling in the autumn of 1985. We don't know the technique and materials used.

The patient has no complaints, and the first radiographic investigation is made 12 years later, in 1997 (fig. 1). Intraoral radiograph reveals teeth with compromised endodontic treatment. Overextension of filling material into the surrounding bone tissues with a resorption around it on 3-4 $\mathrm{mm}$ apically to 31 is confirmed. Voids are present in the root filling and between root filling and root canal walls, no consistent taper is present from the orifice to the apex. Root canals are over-filled and short-filled in the same time. There is a loss of alveolar bone between 31 and 41 .

The root canal filling is not placed within $0.5-2 \mathrm{~mm}$ of the radiographical apex to prevent post-treatment disease (8). Length, shape and density of root canal material are inadequate. The length of an ideal fill should be from the canal's apical minor constriction to the canal's orifice unless a post is planned, the shape 
of the completed case is somewhat dependent on the instrumentation technique being used, and voids should not be visible on the radiographic image (9).

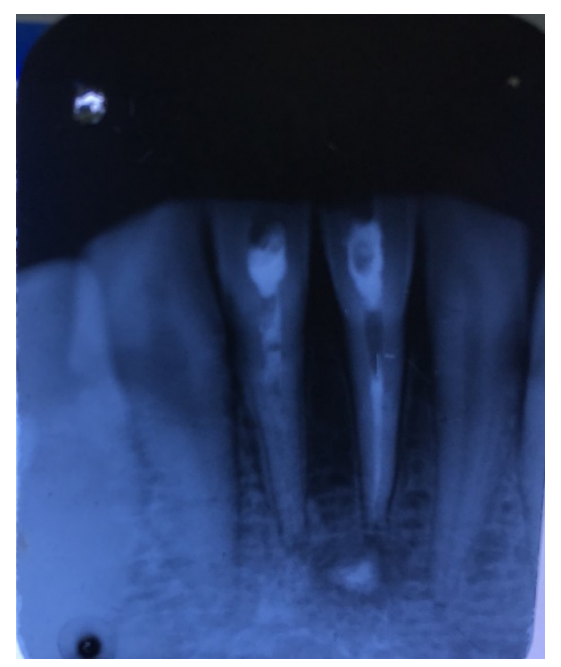

Fig 1. 12 years after the treatment -1997

An endodontic revision with apical surgery is recommended, but no treatment is executed, and a new X-ray is made 3 years later, in April 2000, 15 years after the initial treatment (fig.2). No big difference is observed.

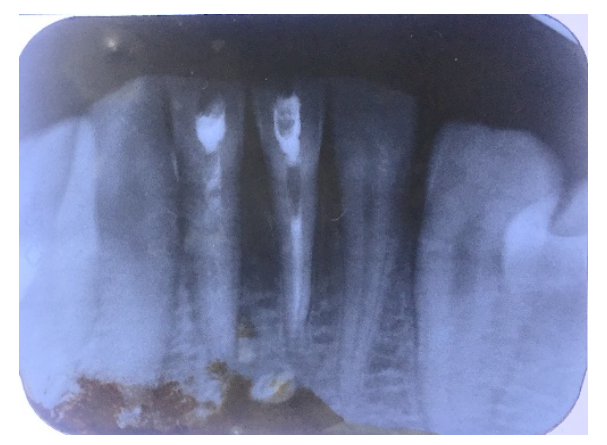

Fig 2. 15 years after the treatment -2000

The patient has no complaints and the next X-rays are made in 2006. The alveolar bone is in good condition. 21 years after the initial treatment a very small amount of root canal filling is present. Osteogenesis is stimulated and most of the filling is gone, due to bone metabolism (fig.3). We can also see an elimination of root canal filling material from the root of 31 . 


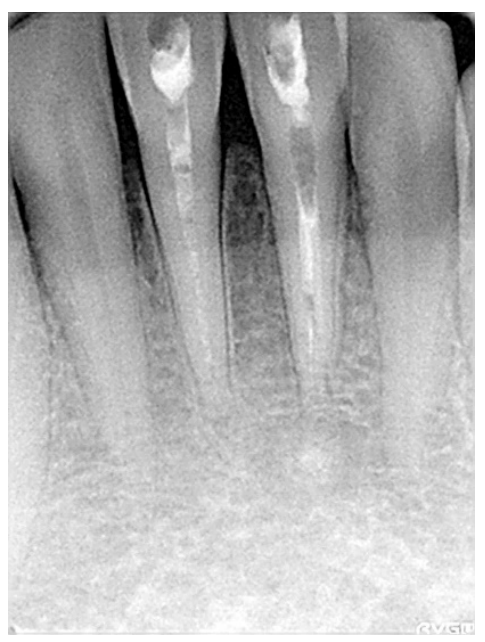

Fig 3. 21 years after the treatment -2006

In the radiographic examination made in 2012, 27 years after the treatment, we see total elimination of root canal filling in the bone (fig.4). We can see the continuing elimination of the root canal filling in the apical third of 31 .

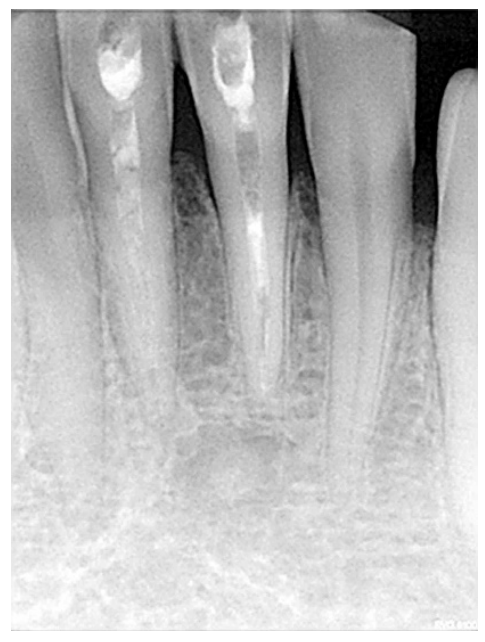

Fig 4. 27 years after the treatment -2012

In 2019, 34 years after the treatment, there is no root canal filling in the bone (fig.5). Root canal filling of 31 is greatly decreased in the tooth canals.

The patient has not received any treatment and has never had any complaints during these 34 years. The teeth are still restored with the temporary obturation from 1985 (fig 6.). Tooth discoloration, which is common following root canal treatment, is observed. 


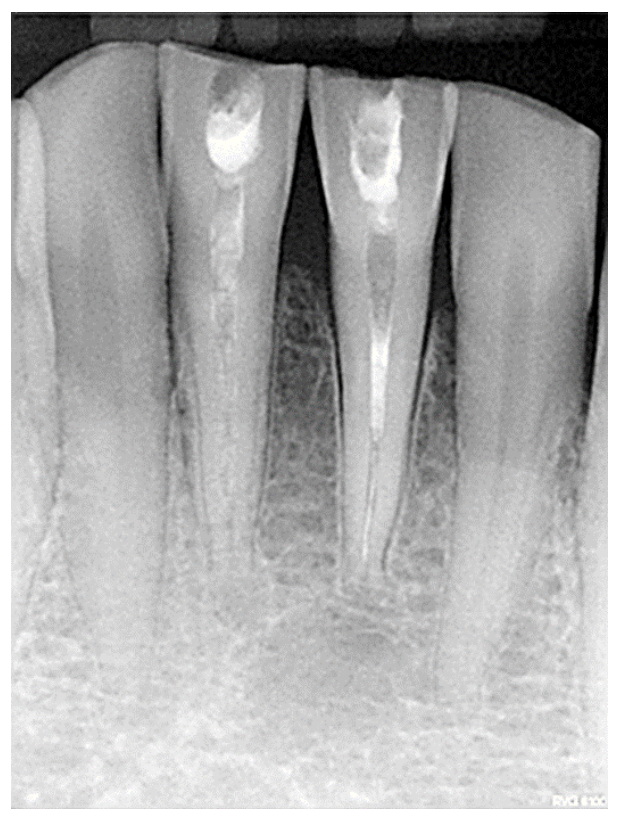

Fig 5. 34 years after the treatment -2019

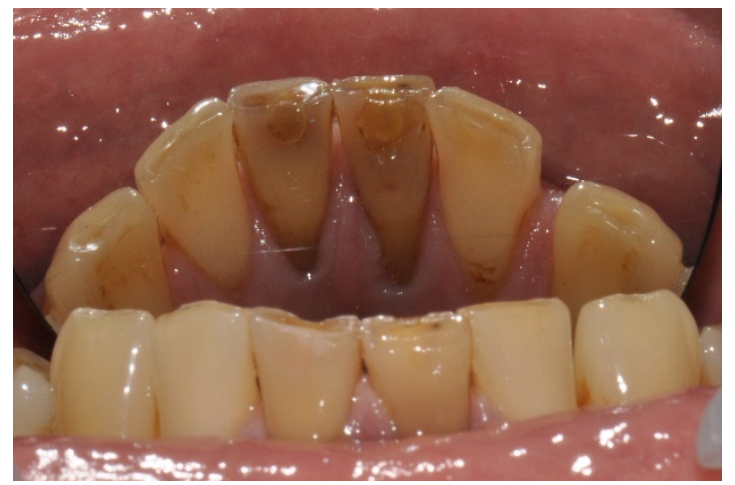

Fig 6. 34 years after the treatment

\section{Discussion}

It has been well established that overfilling should be avoided during obturation. Overfilling of the root canal is indicated only in those cases which will be followed by apicoectomy, when the foreign material will be removed. Accidental overfilling may occur with soft or hard material (pastes, cements, gutta-percha, silver cones). Overfilling may cause an unnecessary mechanical and chemical irritation, which hinders repair of the periapical tissue and thus diminishes the probability of a successful prognosis. The patient may experience sharp, localized postoperative pain, which gradually disappears (10). 
Histological analysis reveals that zinc-oxide-eugenol-based filling material supports inflammatory reaction in the tissue, but bone tissue shows statistically lower inflammatory response in comparison to soft tissue (11).

If this happens with formaldehyde-containing paste, the formaldehyde will be transformed into a harmless substance. The overfill material will be gradually absorbed and the end result is most often good, without long-term complications (2).

The quality of the obturated canals reveals that more than half of the samples (53.4\%) are adequate in length, while only $13.1 \%$ are adequate in density and $14.2 \%$ are adequate in taper. Anterior as well as single-rooted teeth have significantly better quality than posterior and multi-rooted teeth, respectively. In the mandible, central incisors have the highest quality along all criteria followed by second molars and then first molars. It can be noted that quality of length is most adequate in one-canal roots (81.5\%) followed by mesial roots (80.0\%) and then distal roots (65.0\%) (12)

\section{Conclusion}

We assume that in 1985, the amount of the overfilled root canal paste is quite large, observing its complete absorption over a period of more than 25 years. At the 34-th year, we see only a slight lightening and a complete lack of filling material in the bone.

The resorption of the filling from the root canal of 31 is also interesting. On the first radiograph, a wellobtured root canal is visible. Over the years, there has been a decrease in the amount of filling. We can clearly see its large decrease for 34 years, with the canal already looking almost empty, as if the filling is leaking.

Over time, root canal filling is eliminated not only from the bone, but from the root canals also.

\section{Acknowledgements}

In 1985, while carrying out the endodontic treatment, the doctor insists that the patient should become a dentist. And I am!

\section{References}

1. Friedman S, Mor C, The success of endodontic therapy: healing and functionality, J Calif Dent Assoc, 2004, 32 (6): 493-503.

2. Kwak Y, Choi J, Kim K et al. The 5-Year Survival Rate of Nonsurgical Endodontic Treatment: A Population-based Cohort Study in Korea. J Endod. 2019 Oct;45(10):1192-1199.

3. Salehrabi R, Rotstein I. Endodontic treatment outcomes in a large patient population in the USA: an epidemiological study. Journal of Endodontics. 2004, 30 (12): 846-850. 
4. Zadik $\mathrm{Y}$, Sandler $\mathrm{V}$, Bechor $\mathrm{R}$ et al, Analysis of factors related to extraction of endodontically treated teeth, Oral Surg Oral Med Oral Pathol Oral Radiol Endod, 2008, 106 (5): e31-35

5. Neaverth EJ. Disabling complications following inadvertent overextension of a root canal filling material. J Endod, 1989; 15: 135-139.

6. Köseoğlu BG, , Tanrikulu S, , Sübay RK et al. Anesthesia following overfilling of a root canal sealer into the mandibular canal: a case report. Oral Surg Oral Med Oral Pathol Oral Radiol Endod 2006; 101: 803-806.

7. Jo-Eun Kim, Jun-Bum Cho, Won-Jin Yi et al, Accidental overextension of endodontic filling material in patients with neurologic complications: a retrospective case series, Dentomaxillofacial Radiology, 2016, 45:5

8. European Society of E. Quality guidelines for endodontic treatment: consensus report of the European Society of Endodontology. Int Endod J. 2006;39:921-930.

9. $\mathrm{AAE}$ Colleagues for Excellence Newsletter. 2009, DOI: https://doi.org/10.3768/rtipress.2009.rr.0005.0905.

10. D.W. Nitzan, A. Stabholz, B. Azaz, Concepts of accidental overfilling and over instrumentation in the mandibular canal during root canal treatment, Journal of Endodontics,1983, Volume 9, Issue 2, $81-85$.

11. Tarasenko SV, Grigor'janc LA, Morozova EA et al. Histological evaluation of inflammatory response to zinc-oxide-eugenol filling materials in a soft and bone tissues. Stomatologiia (Mosk). 2019;98(5):11-14.

12. Al-Anesi, M.S., AlKhawlani, M.M., Alkheraif, A.A. et al. An audit of root canal filling quality performed by undergraduate pre-clinical dental students, Yemen. BMC Med Educ, 2019, 19, 350

\section{Corresponding author:}

Vladimir Panov, DMD, PhD, DSc,

Professor, Head of Department of Conservative Dentistry

and Oral Pathology, Faculty of dental medicine,

Medical University - Varna,

email: vladimir.panov@mu-varna.bg 